\title{
Clinicoradiographic Evaluation and Feasibility of Dental Implant in Infected Dentoalveolar Socket
}

\author{
${ }^{1}$ Ravi S Batra, ${ }^{2}$ Dayashankara Rao JK, ${ }^{3}$ Vijaylaxmy, ${ }^{4}$ Abhishek Kukreja \\ ${ }^{1}$ Professor, Department of Oral and Maxillofacial Surgery, SGT Dental College and Hospital, Gurgaon, Haryana, India \\ ${ }^{2}$ Professor and Head, Department of Oral and Maxillofacial Surgery, SGT Dental College and Hospital, Gurgaon, Haryana, India \\ ${ }^{3}$ Reader, Department of Oral and Maxillofacial Surgery, SGT Dental College and Hospital, Gurgaon, Haryana, India \\ ${ }^{4}$ Postgraduate Student, Department of Oral and Maxillofacial Surgery, SGT Dental College and Hospital, Gurgaon, Haryana, India
}

Correspondence: Ravi S Batra, Professor, Department of Oral and Maxillofacial Surgery, SGT Dental College and Hospital Budhera, Gurgaon, Haryana, India, e-mail: ravisbatra@yahoo.co.in

\section{ABSTRACT}

Purpose: To describe a protocol for immediate placement of endosseous implant into debrided infected dentoalveolar socket.

Patients and methods: A total of 21 implants were placed in 10 patients. The immediate placement protocol emphasized the meticulous debridement of the infected tissue in combination with periapical ostectomy of socket. Guided bone regeneration was accomplished to support bony healing of alveolar defects surrounding implantation site. Pre- and postsurgical antibiotic therapy was administered.

Results: All implants, but one, were osseointegrated after 6 months follow-up and were functional 6 months postoperatively. One implant was mobile on exposure after 6 months and was removed.

Conclusion: Successful immediate implantation in debrided infected alveolus depends on complete removal of all contaminated tissue and the controlled regeneration of alveolar defect.

Keywords: Immediate implants, Infected sockets.

\section{INTRODUCTION}

According to conventional protocol, implant placement should be delayed up to one year after tooth extraction to allow for complete alveolar bone healing. ${ }^{1,2}$ But the disadvantage during socket healing coupled with an added surgical stage and alveolar ridge resorption after extraction, which considerably reduces the bone volume and compromise the favorable positioning of the implant, required for optimal restoration. ${ }^{4}$

The conventional protocol has been challenged in certain decades by reducing the time between extracting a tooth and placing and loading the implant. An implant placed in fresh extraction socket was noted as immediate implant and an implant placed within 8 week after tooth extraction was called as an immediate delayed implant. An implant placed later than 2 months was called as delayed implant.

The advantage of immediate implant is, it reduces the time period and number of surgical interventions, high chances of implant survival rate (ranging from 93.6\% to $100 \%)^{5}$ and also improved esthetic maintenance of hard and soft tissue at extraction site and higher patient satisfaction as compared with delayed implant placement. ${ }^{3,4}$

Periodontally compromised teeth that are indicated for extraction are involved with infectious condition which conventionally contraindicates the immediate replacement with endosseous dental implants. Updated review suggested that this procedure should be avoided in the presence of periapical and periodontal pathosis. ${ }^{5}$ Barzilay reported that the teeth with periapical pathosis or active periodontal disease are not a candidate for immediate implant, Becker and Becker agrees with this report. ${ }^{6}$

Recent experimental studies in animals have corroborated this clinical experience and shown that socket debridement and prophylactic antibiotics create adequate condition for bone remodeling process around immediate implant placed into infected site. ${ }^{7-9}$

Recent publications advocated the use of nonresorbable barrier technique to aid in healing of implant placed into extraction sockets. Becker et al reported the use of augmentation material around immediate implants in dogs. Their study showed that the augmentation material significantly enhanced bone formation at the site of immediate implantation.

Based on these observations, we in our center at SGT Dental College and Hospital had planned to conduct a study on immediate implant placement of endosseous implant into debrided infected dentoalveolar socket. Our objective was to describe the procedure and to report our experience with 20 implants.

\section{PATIENTS AND METHODS}

A total of 21 implants were immediately placed in debrided infected sites in 10 patients by the treatment protocol detailed 
below. All patients receiving the outlined treatment had been given a detailed explanation and gave their informed consent for the procedure.

\section{Inclusion Criteria}

- Maxillary and mandibular teeth that are indicated for extraction because of presence of periapical and periodontal pathosis.

\section{Exclusion Criteria}

- Unmotivated patient

- Medically compromised patient

- Habits and behavioral consideration

i. Chronic smoking

ii. Tobacco chewers

iii. Parafunctional habits

iv. Substance abuse (e.g. alcohol, drugs)

- Patient with hypersensitivity to implant material

- Patient with poor oral hygiene.

\section{Treatment Protocol}

A patient was initiated on a daily dose of $500 \mathrm{mg}$ amoxicillin and $400 \mathrm{mg}$ of metronidazole, 8 hourly, orally, 2 days prior to surgical procedure. Strict aseptic protocol was followed. Intraoral and extraoral scrubbing and draping was done.

All the procedures were performed under local anesthesia, teeth were extracted atraumatically. Every attempt was made to have minimal trauma to alveolus during extraction (Figs 1 and 2). After extraction of teeth, the extraction sockets were thoroughly debrided and curetted to remove any granulation tissue and necrotic bone from the socket. Length of the root of tooth and diameter of radiolucency present at the apex of tooth was then measured and $2 \mathrm{~mm}$ is then added to the above value so as to determine the length of the implant to be inserted. Mesiodistal width of the tooth is also measured to determine the width of the implant (Fig. 3). Drills were used in proper sequence according to the manufacturer's instructions (Fig. 4). The final drill should engage both buccal and lingual cortical plate (if possible) so that a good primary stability can be achieved by implant. After the osteotomy of the socket was completed up to the desired length and width, implant was placed into the socket into its final seating position. After the implant placement was done in the infected socket, the defects that were present in the socket wall and the implant surface were grafted using hydroxyapetite with ß-tricalcium phosphate. GTR membrane was then placed over the grafted site so as to prevent soft tissue in growth between the implant and the bone (Fig. 5). Primary (water tight) closure of the soft tissue is usually recommended so as to prevent the dislodgement of graft material. If primary closure is not

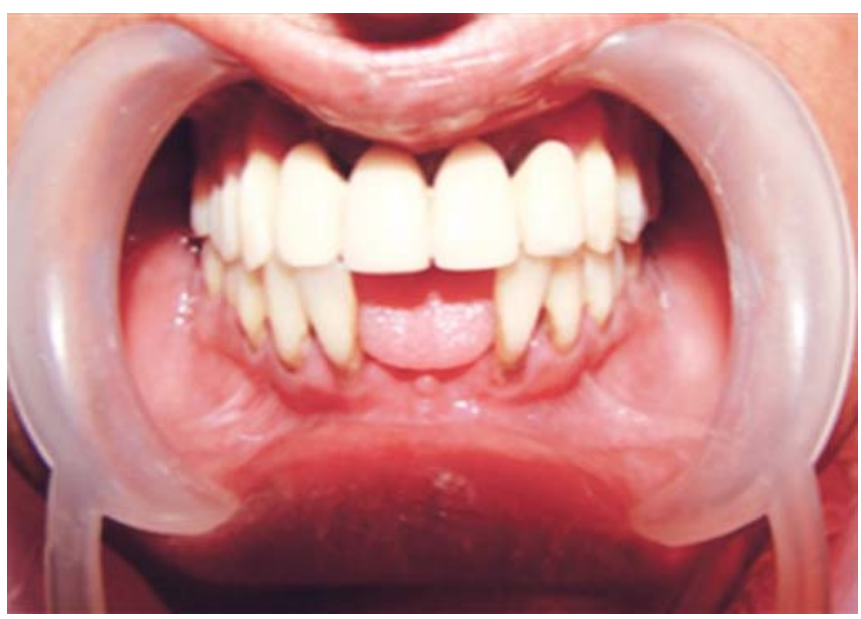

Fig. 1: Preoperative picture

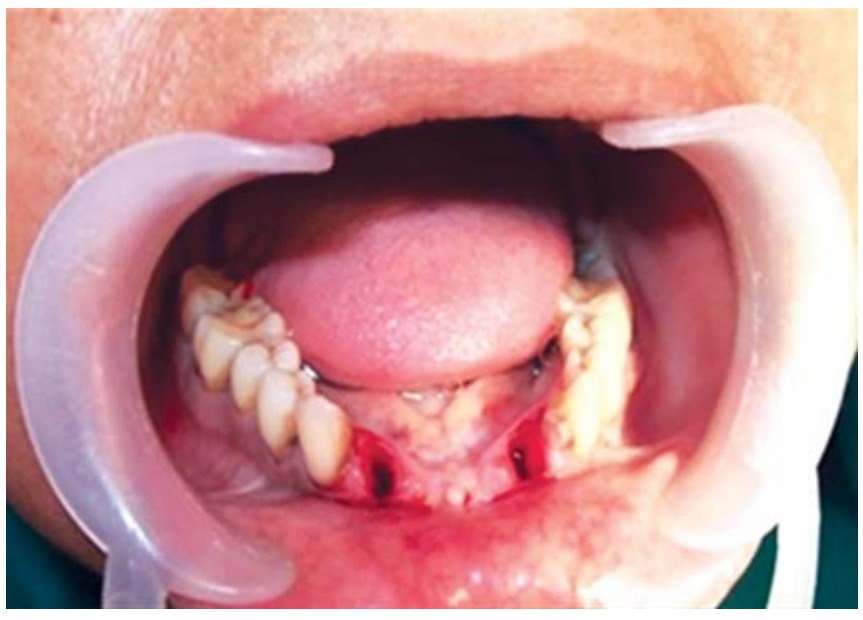

Fig. 2: After extraction of 33,43

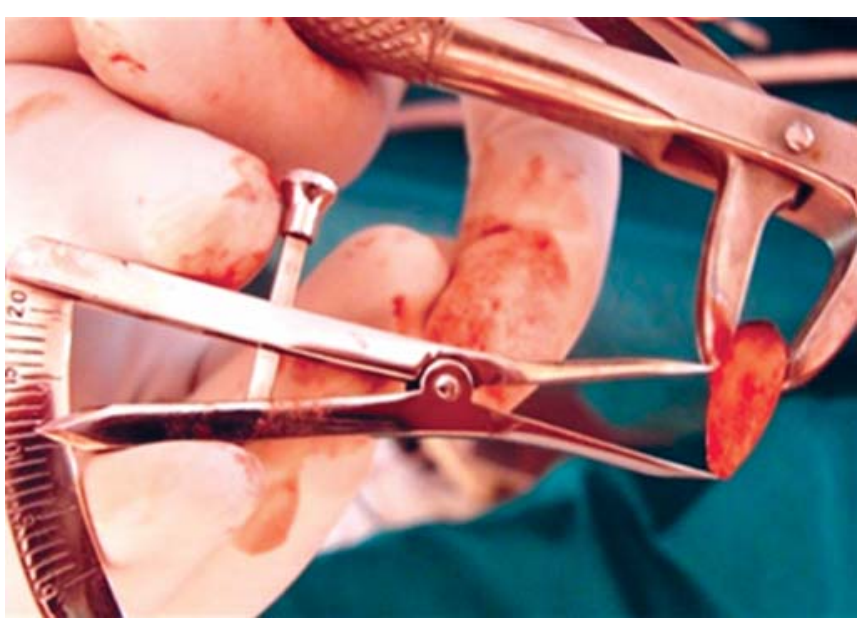

Fig. 3: Measuring the root length of extracted tooth

possible, use of vertical incision or horizontal scoring of the periosteum or both were used for flap manipulation to achieve the primary closure (Fig. 6).

\section{RESULTS}

On evaluation, we found that healing was uneventful in all implants and there was no sign of inflammation, infection 


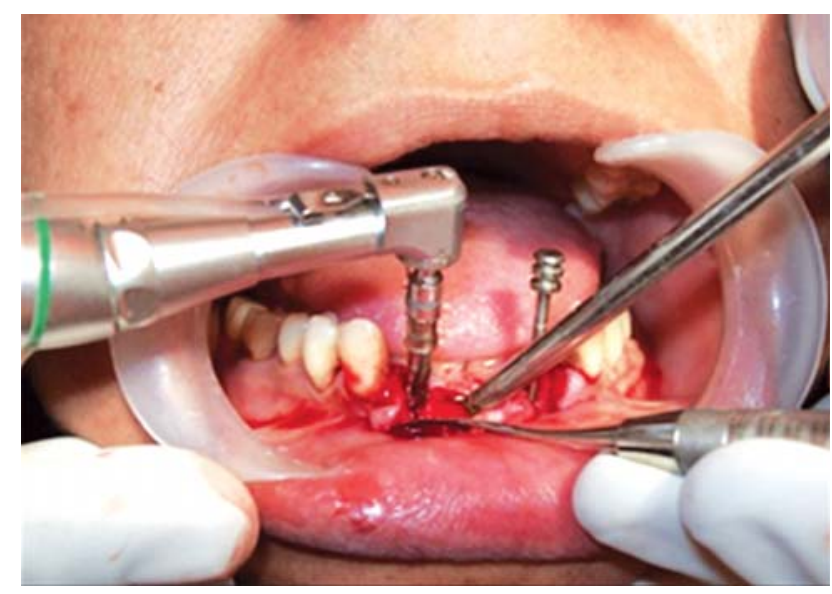

Fig. 4: Drilling the osteotomy

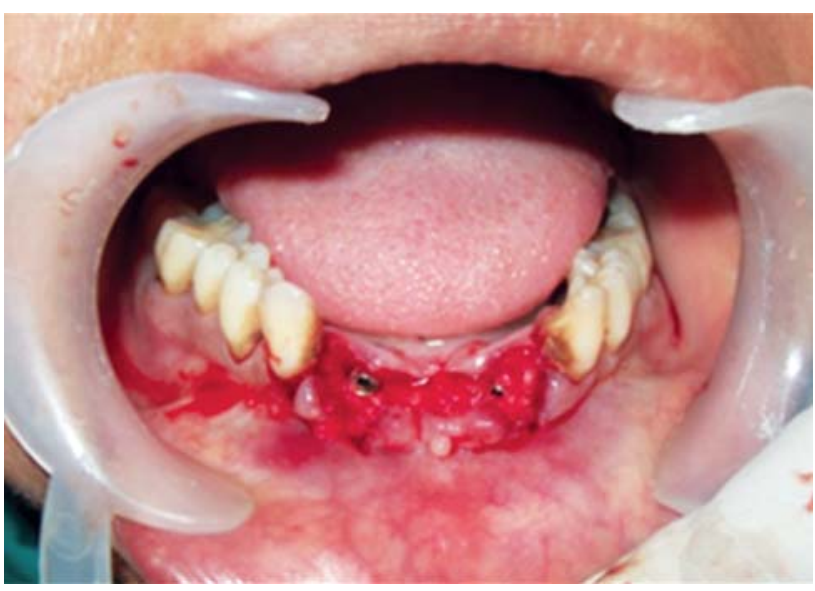

Fig. 5: Implants inserted

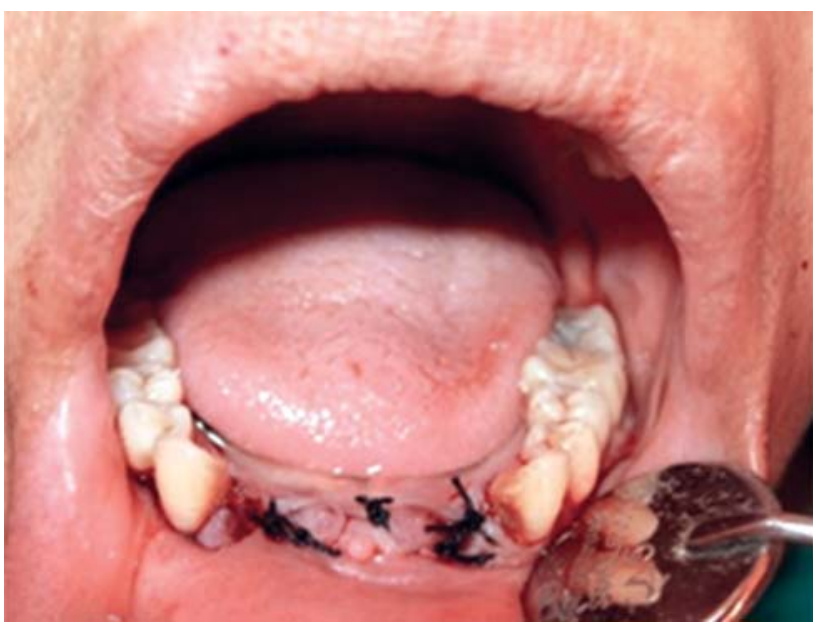

Fig. 6: Site sutured

or peri-implant radiolucency. All the implants were firm on re-exposure, except one which was placed in the region of 44. Implant was then removed and the site was curetted and left for healing by secondary intention.

Out of 21 implants we have placed, 20 implants achieved osseointegration and are loaded successfully. In our study overall success rate is $95 \%$.

\section{CASE REPORTS}

\section{Case 1 (Figs 7 and 8)}

A 30-year-old male patient reported with acute pain in lower left mandibular region. On intraoral palpation, 35 and 36 was tender on percussion and on radiological examination both the teeth had periapical radiolucency. Treatment plan consisted of extraction of 35 and 36 and implant-supported restoration. Six months after surgical procedure implants were re-exposed after confirmation of osseointegration, radiologically and clinically both the implants were prosthetically rehabilitated.

\section{Case 2 (Figs 9 and 10)}

A 15-year-old male complained of pain in his lower right back region. On intraoral examination, root stumps, present in the region of 36 and 46 was tender and on radiological examination both the teeth had periapical radiolucency. Treatment plan consisted of extraction of 36 and 46 and implant-supported restoration. Six months after surgical procedure implants were re-exposed after confirmation of osseointegration, radiologically and clinically both the implants were prosthetically rehabilitated.

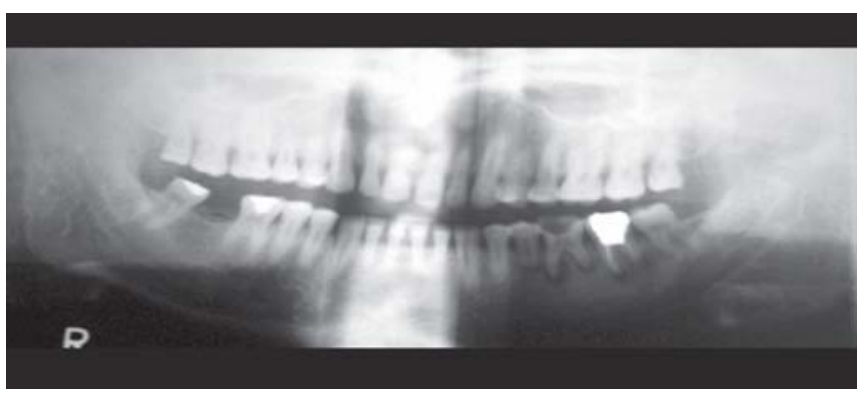

Fig. 7: Preoperative (Case 1)

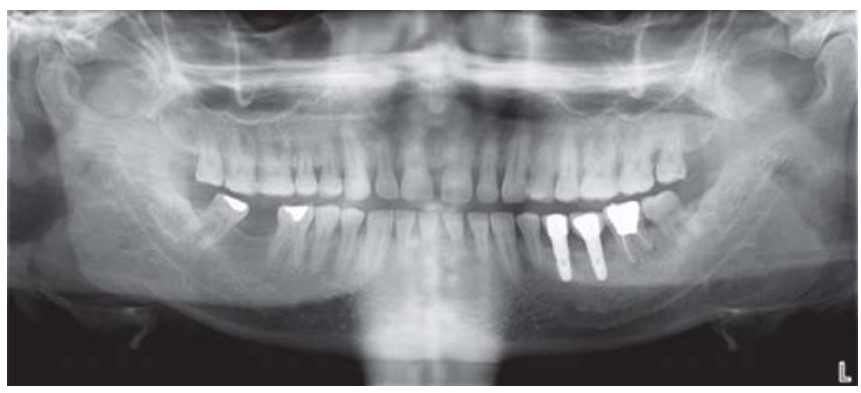

Fig. 8: Postoperative (Case 1)

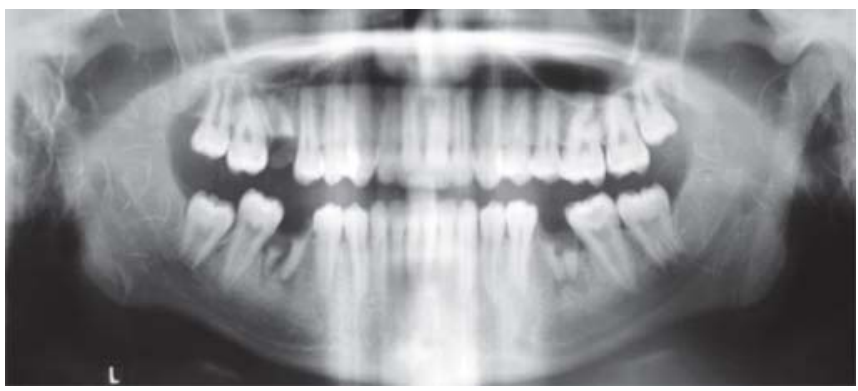

Fig. 9: Preoperative (Case 2) 


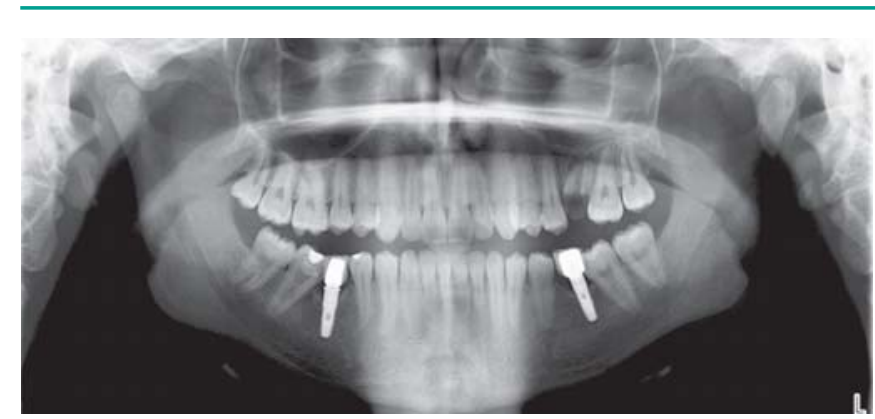

Fig. 10: Postoperative (Case 2)

\section{DISCUSSION}

According to conventional protocol, implant placement should be delayed up to one year after tooth extraction to allow complete alveolar bone healing, ${ }^{2,3,10}$ which is one of the major disadvantage of implant surgery.

Many studies on immediate placement of dental implant in fresh extracted socket had been conducted with success of almost above $90 \%$ in all the studies and is now become a well-establish protocol. ${ }^{10,11}$

Most of the teeth that were extracted were due to either periodontal or periapical infection and updated review of literature on immediate implant suggests that this procedure should be avoided in the presence of periapical or periodontal pathosis. ${ }^{11}$ Clinical reports have suggested that history of periodontal or endodontic infection is a predictive marker for implant infection and failure. ${ }^{12-15}$ This clinical experience has led most clinicians to avoid the immediate placement of endosseous dental implants at infected site and to consider infection a contraindication for immediate implantation.

The protocol normally followed in this patient situation includes:

- Extraction of tooth

- Debridement

- Bone graft and collagen membrane (if required)

- Placement of implant following a healing period of 6-12 months. ${ }^{16,17}$

Ericksson et $a l^{18}$ suggested that proper antibiotic coverage with immediate implant surgery could minimize the implant failure rate.

Various studies on immediate implant placement in fresh extraction socket confirm that healing and osseointegration were simultaneous process and they appreciate repair phenomenon associated with extraction socket healing and osseointegrated dental implant. They suggested that the conditions associated with the repair of extraction socket may be favorable for integration of dental implants. ${ }^{19}$

As most of our cases have been associated with alveolar defect and adequate bony healing of the alveolar defects vital for the osseointegration of immediately placed implant and for its functional stability, so we strictly maintain a protocol that completely removes the contaminated tissue and allows successful bone regeneration of the previously infected alveolus.
As our main aim is to achieve osseointegration of implants and as there are high chances in our cases for soft tissue ingrowth in between implant surface and bone because of presence of alveolar defect, we have filled the defect with allograft and covered the grafted site using barrier technique. $^{20-22}$ These materials will prevent connective tissue ingrowth between implant and bony wall that might interfere with osseointegration of implant. ${ }^{23}$ We have used allogenic bone graft material ( $\beta$-tricalcium phosphate) and GTR membrane to prevent soft tissue ingrowth between implant and bone to achieve osseointegration.

\section{CONCLUSION}

In this study, we have challenged the conventional concept of not placing the implant in infected socket and argued that under a controlled procedure and by following strict protocol, implants can be successfully introduced into debrided infected dentoalveolar socket immediately after extraction.

\section{REFERENCES}

1. Carl E Misch. Contemporary implant dentistry (3rd ed).

2. Anneroth G, Headstrom KG, Kjellman O, Kondell PA, Nordenram A. Endosseous titanium implant in extraction sockets-An experimental study in monkey. Int J Oral Surg 1985;14:50-54.

3. Pierluigi Avvanzo, Dominico Ciavarella, Andrea Avvanzo, Nicola Giannone, Mauro Carella, Lorenzo Lo Muzio. Immediate placement and temporization of implants: Three to five-year retrospective results. J of Oral Implantology 2009;3:136-42.

4. John L Krump, Baron G Barnett. The immediate implant: A treatment alternative. Int J Oral Maxillofac Implants 1991;6: 19-23.

5. Izchak Barzilay, Gerald N Graser, Bejan Iranpour, Howard M Proskin. Immediate implantation of pure titanium implants into extraction sockets of macaca fascicularis (part I): Clinical and radiographic assessment. Int J Oral Maxillofac Implants 1996;11:299-310.

6. Arthur B Novaes Jr, Arthur B Novaes. Immediate implant placed into infected sites: A clinical report. Int J Oral Maxillofac Implants 1995;10:609-13.

7. Arthur B Novaes Jr, Guaracilei M Vidigal, Arthur B Novaes, Marcio FM Grisi, Sergio Polloni, Adalberto Rosa. Immediate implant placed into infected sites: A histomorphometric study in dogs. Int J Oral Maxillofac Implants 1998;13:422-27.

8. Arthur B Novaes Jr, Andrea M Marcaccini, Sergio LS Souza, Mario Taba Jr, Marcio FM Grisi. Immediate placement of implants into periodontally infected sites in dogs: A histomorphometric study of bone-implant contact. J Oral Maxillofac Implants 2003;18:391-98.

9. Cyril I Evian, Robert Emling, Edwin S Rosenberg, Jonathan A Waasdrop, Wendy Halpern, Shalin Shah. Retrospective analysis of implant survival and influence of periodontal disease and immediate placement on long-term results. Int J Oral Maxillofac Implants 2004;19:393-98.

10. Douglas Allen Atwood. Some clinical factor related to rate of resorption of residual ridges. J Pros Den 1962;12:441-50.

11. Nardy Casap, Chassiel Zeltser, Alon Wexler, Eyal Tarazi, Rephael Zeltser. Immediate placement of dental implant into debrided infected dentoalveolar sockets. J Oral Maxillofac Surg 2007;65:384-92. 
12. Schwartz-Arad, Chaushu G. The ways and wherefores of immediate placement of implants into fresh extraction sites: A literature review. J Periodontal 1997;68(10):915-19.

13. Barzilay I. Immediate implants: There current status. Int J Prosthodont 1993;6(2):169-77.

14. Becker W, Becker BE. Guided tissue regeneration of implants placed into extraction socket for their implant dehiscences: Surgical technique and case report. Int J Periodontics Restorative Dent 1990;10(5):376-87.

15. Werbitt MJ, Goldberg PV. The immediate implant bone preservation and bone regeneration. Int J Periodontics Restorative Dent 1992;12(3):206-17.

16. Lundgren D, Nyman S. Bone regeneration in two stages for retention of implants: A case report. Clin Oral Implants Res 1991;2(4):203-07.

17. Wilson TG Jr. Guided tissue regeneration around dental implants in immediate and recent extraction sockets: Initial observation. Int J Periodont Rest Dent 1992;12(3):185-93.

18. Lekholm U, Adell R, Lindhe J, Branemark PI, Eriksson B, Rockler B, et al. Marginal tissue reaction at osseointegrated titanium fixtures: A cross section retrospective study. Int J Oral Surg 1986;15(1):53-61.
19. Yukna RA, et al. Placement of hydroxyapatite coated implants into fresh or recent extraction sites. Dent Clin North Am 1992;36:97-115.

20. John C Minichetti, D’Amore JC, Hong AY. Three-year analysis of tapered screw-vent implants placed into extraction socket grafted with mineralized bone allograft. J of Oral Implantology 2005;31:283-93.

21. William Becker, Robert Schenk, Kenji Higuchi, Ulf Lekholm, Bruton E Becker. Variation in bone regeneration adjacent to implant augmented with barrier membrane alone or with demineralized freeze dried bone or autologous grafts: A study in dogs. Int J Oral Maxillofac Implants 1995;10:143-54.

22. Miguel Penorrocha, Maria Penarrocha, Berta Garcia-Mira, Carolina Larrazabal. Extraction of impacted maxillary canines with simultaneous implant placement. J Oral Maxillofac Surg 2007;65:2336-39.

23. William Becker, Christer Dhlin, Bruton E Becker, Ulf Lekholm, Daniel Van Steenberghe, et al. The use of e-PTFE barrier membranes for bone promotion around titanium implants placed into extraction sockets: A prospective multicenter study. Int J Oral Maxillofac Implants 1994;9:31-40. 